ISSN: 0210-1696

DOI: https://doi.org/10.14201/scero2021523141161

\title{
CIENCIA INCLUSIVA, CINE Y CREATIVIDAD: HERRAMIENTAS PARA MEJORAR LA CALIDAD DE VIDA DE LAS PERSONAS CON DISCAPACIDAD INTELECTUAL
}

\section{Inclusive Science, Film and Creativity: Tools to Improve the Quality of Life of People with Intellectual Disabilities}

Diego Ortega-Alonso

Universidad de Jaén

info@diegortegalonso.com

M. ${ }^{a}$ Elvira de CASTRO-López

AFAMP

direccion@afamp.com

Recepción: 5 de diciembre de 2019

Aceptación: 19 de octubre de 2020

Resumen: Este artículo tiene como objeto revisar la importancia del desarrollo de acciones de innovación social y creatividad en el ámbito del ocio para las personas con discapacidad intelectual en particular, y para la sociedad en general, a través de un estudio de caso: el proyecto PDICIENCIA. Este aborda de forma proactiva la plena inclusión de las personas con discapacidad intelectual a través de acciones innovadoras que fomentan la cultura científica, tecnológica y de la innovación entre públicos habitualmente alejados de estas áreas. Además, empodera a las personas con discapacidad intelectual para dotarlas de unas habilidades profesionales y sociales. Veremos cómo su participación activa en actividades de creatividad, divulgación científica o producción cinematográfica, implantadas a través de los planes de atención individualizados de la Asociación de Familiares y Amigos de Personas con Discapacidad Intelectual (AFAMP) en la Residencia Pedro Gámez de Bailén (Jaén), puede incidir en la mejora de su calidad de vida. 
Palabras Clave: creatividad; discapacidad intelectual; ciencia inclusiva; innovación social; calidad de vida.

AвstRAct: The purpose of this article is to review the importance of the development of actions of social innovation and creativity, in leisure time, with people with intellectual disabilities in particular, and for society in general, through a case study: PDICIENCIA. It is a project that proactively seeks the full inclusion of people with intellectual disabilities through innovative actions to promote scientific, technological and innovation culture among audiences usually removed from these areas. In addition, it empowers people with intellectual disabilities to equip them with professional and social skills. We will see how their active participation in creativity, scientific dissemination or film production activities, implemented through the individualized care plans of the Association of Family and Friends of People with Intellectual Disabilities (AFAMP) in the Pedro Gámez Residence, from Bailén (Jaén, Spain), can affect improving their quality of life.

KEYWORDS: creativity; intellectual disability; inclusive science; social innovation; quality of life.

\section{Introducción}

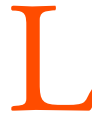

A EXISTENCIA DE UN PAPEL TRADICIONAL de la práctica artística en el "tratamiento” terapéutico de las personas con discapacidad intelectual cuenta con numerosos ejemplos y publicaciones sobre prácticas inclusivas fundamentadas en las artes plásticas o la arteterapia (Alonso, 2016; Alonso, 2018; Ballesta et al., 2011; Campos, 1995; Cárdenas et al., 2017; García, 2013; Jiménez et al., 2013), encuadradas en su mayor parte en los criterios de normalización de Bank-Mikkelsen (1975) o García $(1988)^{1}$. El trabajo artístico brinda a estas personas oportunidades de expresión, desempeño y conexión con la gente, estimulando el interés y el debate público sobre sus capacidades (Hall, 2010).

Las personas adultas con discapacidad intelectual, sin embargo, han permanecido alejadas, en términos generales, de los procesos educativos interdisciplinares ligados a la implementación de metodologías de carácter creativo e innovador. No ocurre lo mismo en el ámbito de la integración de otros colectivos, como, por ejemplo, el caso de personas en riesgo de exclusión social (Moreno y Martínez, 2019), en el de la integración educativa o en las comunidades, donde las prácticas artísticas colaborativas de carácter artivista suelen estar centradas en la transformación social, dejando en un segundo plano los conceptos puramente formales y la estética (Aladro Vico et al., 2018).

1 "Normalizar no es pretender convertir en normal a una persona deficiente, sino reconocerle los mismos derechos fundamentales que los demás ciudadanos del mismo país y de la misma edad. Normalizar es aceptar a la persona deficiente, tal como es, con sus características diferenciales, y ofrecerle los servicios de la comunidad para que pueda desarrollar al máximo sus posibilidades, y vivir una vida lo más normal posible” (García, 1988, p. 69). 
Según el estudio sobre el impacto de la Convención Internacional sobre los Derechos de las Personas con Discapacidad en la legislación educativa española elaborado por CERMI (2011), la segregación educativa se instauró como un modelo para facilitar una educación de mayor calidad al centrarse en grupos homogéneos. La dignidad inherente a todo ser humano no les fue reconocida hasta hace pocas décadas, ya que antes ni siquiera eran consideradas dignas de ser educadas, cuando no se les eliminaba o excluía completamente de la vida social (Alonso y De Araoz, 2011).

El movimiento asociativo está comenzando a poner en marcha prácticas que utilizan la innovación social como eje de transformación de los servicios hacia la Calidad de Vida (CDV), enmarcadas en la estrategia definida por el movimiento Plena inclusión a favor de las personas con discapacidad intelectual o del desarrollo y sus familias (Tamarit, 2015). Es este un tipo de innovación disruptiva (Christensen et al., 2015) que presupone una transformación organizacional profunda (Martínez-Tur y Peñarroja, 2012) y un cambio de paradigma que incide en la atención a la persona (Schalock y Verdugo, 2007). El trabajo que realizan las entidades dedicadas a generar los apoyos y las oportunidades necesarias para que las personas con discapacidad y sus familias puedan desarrollar una vida plena, apostando por su inclusión en todos los ámbitos, incluye la educación. Esta debe abordar aspectos de carácter innovador que vayan más allá de las estrategias que se utilizan en ámbitos educativos normalizados, y el empoderamiento y fomento de la creatividad deben tener un papel fundamental. En el presente artículo se exponen algunas de las actividades desarrolladas en este sentido en la Residencia y Centro de Día Ocupacional (CDO) Pedro Gámez de Bailén (Jaén), que gestiona AFAMP, y que, en su conjunto, han influido exponencialmente en la educación, la inclusión, el desarrollo de capacidades y la mejora de la CDV de estas personas.

Los criterios de valoración se apoyaron en las dimensiones de CDV sobre las que trabajar en la intervención con cada persona, de acuerdo con el Modelo de CDV Individual de Schalock y Verdugo (2007). Los modelos de trabajo actuales de AFAMP se fundamentan en el apoyo conductual positivo (Goñi et al., 2012), así como en el apoyo activo (Rodríguez et al., 2016), en el modelo de transformación de servicios (Tamarit, 2015) y la Planificación Centrada en la Persona (Carratalá et al., 2018), los cuales se sitúan principalmente en las capacidades que tienen las personas, los apoyos que se necesitan para desarrollarlas y las oportunidades que se generan de forma individualizada. Se pone el énfasis en las capacidades que tiene la persona y en que es más capaz cuantas más oportunidades y apoyos se le facilitan².

\section{El Proyecto PDICIENCIA}

AFAMP lleva desde el año 1987 dedicándose a facilitar apoyos y oportunidades a las personas con discapacidad intelectual y a sus familias en la localidad de Bailén y provincia de Jaén, promoviendo actuaciones dirigidas a su inclusión participativa,

2 Programación Residencia Pedro Gámez de Bailén, p. 4. 
así como a su sensibilización y promoción. En el Plan Estratégico de AFAMP para el período 2019-2021, se recoge la atención hacia el protagonismo de cada persona en su vida. Para ello, se tienen en cuenta el trato individualizado, las necesidades, expectativas, deseos y preferencias para que, junto con las personas más significativas en sus vidas, puedan desarrollar su proyecto de vida. En este proyecto personal se incluyen la creatividad y la innovación como elementos transformadores que llevan a la CDV. Así, desde AFAMP se miden tanto el Índice de Calidad de Vida (ICV) como la satisfacción de cada persona en los servicios de ocio y tiempo libre, concretamente en los talleres de creatividad, de producción cinematográfica y de ciencia inclusiva ${ }^{3}$. En dicho plan estratégico, la asociación asimismo se plantea la necesidad de crear en la sociedad actitudes positivas sobre la discapacidad intelectual ${ }^{4}$, de modo que la ciencia inclusiva se convierte en una herramienta que hace visibles las capacidades de las personas con discapacidad mediante su aportación como comunicadores científicos para todos los públicos, y reporta al entorno la contribución que este ha tenido con el movimiento asociativo de la entidad.

PDICIENCIA 5 surgió en diciembre de 2016 encuadrado en el marco de actividades de ocio y tiempo libre para adultos de la Residencia y CDO Pedro Gámez de Bailén (Jaén). Se trata de un proyecto cuyo objetivo general es difundir el conocimiento y la cultura científica desde una perspectiva de ciencia inclusiva, visibilizando las capacidades de las personas con discapacidad intelectual a través de su participación activa en acciones educativas, científicas y de investigación para lograr su plena inclusión. Para ello, se realizan programas audiovisuales que se distribuyen a través de las redes sociales y la web y que, además, se utilizan como recursos educativos en acciones presenciales. El equipo está formado por un grupo de personas de diferentes capacidades intelectuales y físicas, que trabajan en un ambiente de integración e inclusión en el que se fomentan y apoyan sus distintas capacidades. Las acciones desarrolladas se fundamentan en la aplicación de metodologías del ámbito de la intervención artística y creativa, la innovación social y la comunicación científica.

PDICIENCIA es un proyecto de ciencia abierta (open science), ya que representa un nuevo enfoque en el proceso científico y de investigación basado en el trabajo colaborativo y en las nuevas formas de distribución del conocimiento, utilizando tecnologías digitales y nuevas herramientas de cooperación, que busca hacer la ciencia más creíble (integridad científica), confiable (mejor y más transparente verificación de información), eficiente (evitando duplicar esfuerzos) y más activa en la respuesta a los desafíos sociales ${ }^{6}$. Se postula dentro de los parámetros de la ética hacker, centrada en "el valor de la creatividad combinando la pasión con la libertad [...] donde el beneficio se cifra en metas como el valor social, el libre acceso, la transparencia y la franqueza” (Himanen, 2002, p. 2).

Plan Estratégico AFAMP 2019-2021. Línea 1. Objetivo general 1, objetivos específicos 1, 2, 3. Plan Estratégico AFAMP 2019-2021. Línea 5. Objetivo general 3, objetivo específico, 2.

http://pdiciencia.com

6 Open Innovation. Open Science, Open to the World - a vision for Europe. European Comission. 2016. http://publications.europa.eu/resource/cellar/3213b335-1cbc-11e6-ba9a-01aa75ed71a1.0001.02/DOC_2 


\subsection{Hacia una ciencia accesible e inclusiva}

$\mathrm{El}$ acceso a la ciencia para su goce pleno y en condiciones de igualdad es un derecho fundamental que la Carta Internacional de los Derechos Humanos recoge en su artículo 15 desde 1966, el cual reconoce "el derecho de toda persona a participar en la vida cultural o gozar de los beneficios del progreso científico y de sus aplicaciones, desarrollando y difundiendo la ciencia y la cultura, y fomentando la cooperación”.

El 13 de diciembre de 2006, la Asamblea General de las Naciones Unidas aprobó la Convención Internacional sobre los Derechos de las Personas con Discapacidad, entrando en vigor en España el 3 de mayo de 2008. El artículo 1 establece que su fin es "promover, proteger y asegurar el goce pleno y en condiciones de igualdad de todos los derechos humanos y libertades fundamentales por todas las personas con discapacidad, y promover el respeto de su dignidad inherente".

Por su parte, la Agenda 2030 para el Desarrollo Sostenible establece una serie de metas entre las cuales se encuentra la de potenciar y promover la inclusión social, económica y política de todas las personas, independientemente de su edad, sexo, discapacidad, raza, etnia, origen, religión, situación económica u otra condición. Para lograr una inclusión efectiva de las personas con discapacidad intelectual en la sociedad, se torna necesario tomar como punto de partida el reconocimiento de sus habilidades, ya sean de carácter transitorio o permanente, y que fundamente la idea de diversidad sin exclusiones o discriminaciones de ningún orden.

La Encuesta de Percepción Social de la Ciencia y la Tecnología de España, que se realiza bianualmente desde el año 2002, profundiza en el conocimiento de las relaciones entre ciencia, tecnología y sociedad, y analiza la percepción de la ciudadanía sobre los avances científicos y tecnológicos. Los datos recabados por dicha encuesta indican un crecimiento del interés general de la ciudadanía por la ciencia y la tecnología, si bien durante los últimos años el porcentaje prácticamente se mantiene.

\begin{tabular}{|c|c|}
\hline \multicolumn{2}{|c|}{$\begin{array}{c}\text { TABLA 1. Relación de datos de interés por la ciencia } \\
\text { y la tecnología de 2002 a } 2018\end{array}$} \\
\hline Año & Interés mostrado por la ciudadanía en temas científicos y tecnológicos \\
\hline 2004 & $6,9 \%$ \\
\hline 2006 & $9,6 \%$ \\
\hline 2008 & $9,6 \%$ \\
\hline 2010 & $13,1 \%$ \\
\hline 2012 & $16 \%$ \\
\hline 2014 & $15 \%$ \\
\hline 2016 & $16 \%$ \\
\hline 2018 & $16,3 \%$ \\
\hline
\end{tabular}

Fuente: Fundación Española para la Ciencia y la Tecnología (2018). Informe de resultados de Percepción Social de la Ciencia y la Tecnología 2018. Recuperado de: https://icono.fecyt.es/informes-y-publicaciones/ percepcion-social-de-la-ciencia-y-la-tecnologia-en-espana 
Lo cierto es que la ciencia y la tecnología, desde una perspectiva global, siguen distantes con respecto al lugar de interés que ocupan las áreas situadas entre las cinco primeras posiciones del ranking en 2018: medicina y salud (37,9 \%), trabajo y empleo $(31,9 \%)$, educación $(28,8 \%)$, cine, arte y cultura $(24,5 \%)$ y deportes $(24,4 \%)$. Esta encuesta (y es este un dato que nos interesa especialmente) también profundiza en la percepción de la ciudadanía sobre los beneficios aportados por la ciencia y la tecnología para la "Mejora de la Calidad de Vida" de las personas. La imagen que los ciudadanos tienen de la ciencia y la tecnología es positiva, pese a reducirse con respecto a la anterior encuesta de 2016 (que arrojaba un 53,6 \%). Si bien estudios recientes (Alonso-Flores et al., 2018) indican que la mayoría de investigadores (65,4 \%) perciben algún tipo de beneficio después de haber realizado la comunicación de sus resultados de investigación, existen pocas líneas de trabajo que aborden la transferencia del conocimiento en términos inclusivos. Muchos menos aún, aquellos que involucren de forma proactiva a las personas con discapacidad intelectual o del desarrollo en los procesos de creación de la comunicación. Para conseguir este reto, la primera toma de conciencia debe producirse entre la propia comunidad científica. A pesar de que cada vez existe una mayor concienciación acerca de la importancia de la divulgación, sigue habiendo miembros del colectivo que no revierten a la sociedad los resultados de sus investigaciones (García-Frank y Gómez-Heras, 2016).

En el contexto de España, existe un creciente interés en la elaboración de materiales y actividades de divulgación inclusiva, por parte de asociaciones, organismos o entidades dedicados a la investigación y al fomento de la cultura científica (GarcíaFrank et al., 2013; García-Frank et al., 2016; Iglesias et al., 2015; Muñiz et al., 2018; Muñoz-García et. al., 2015; Pérez-Montero, 2019), también a través de la inclusión educativa (véanse proyectos como Incluciencia ${ }^{7}$, Ciencia en el Hogar ${ }^{8}$ o BCN-ConCiencia ${ }^{9}$ ), o mediante la adaptación integral de espacios y colecciones en museos y centros de interpretación, entre los que consideramos especialmente destacable el caso del Museo de la Ciencia de Valladolid a través de su proyecto "Ciencia con diferencia" ${ }^{10}$. Este aglutina todas las acciones de accesibilidad del museo, entre las que se encuentran la señalización de los espacios en pictogramas y braille, o la adaptación de textos en lectura fácil (Rodríguez, 2017). Otro ejemplo reciente es el proyecto "Ver con las manos, escuchar con la mirada, sentir con los recuerdos y aprender sin limitaciones. El Museo Nacional de Ciencias Naturales, un museo accesible”, presentado oficialmente en mayo de 2019, que cuenta con financiación de la Fundación Española para la Ciencia y la Tecnología.

La segregación educativa supone, entre otras cuestiones, que la percepción social sobre la discapacidad intelectual la sitúe en el extremo opuesto de la excelencia educativa (Alonso y De Araoz, 2011), hasta el punto de que, aislando a estas personas del resto, las convierten en una especie de antagonismo de lo que representa el conocimiento científico. También presupone que la persona con discapacidad intelectual

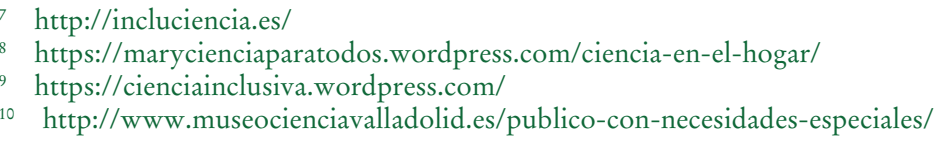


se percibe como un sujeto pasivo que no es capaz de aportar conocimiento (ni, en consiguiente, conocimiento científico). Sin embargo, la apuesta por la integración y la inclusión educativa responde a la percepción de un modelo social que se viene imponiendo en las últimas cuatro décadas y que empodera a las personas con discapacidad, proporcionando la base de un nuevo paradigma transformador que nos lleva directamente a la educación inclusiva, cuyos principales objetivos ${ }^{11}$ vienen a ser:

a) Desarrollar plenamente el potencial humano y el sentido de la dignidad y la autoestima y reforzar el respeto por los derechos humanos, las libertades fundamentales y la diversidad humana.

b) Desarrollar al máximo la personalidad, los talentos y la creatividad de las personas con discapacidad, así como sus aptitudes mentales y físicas.

c) Hacer posible que las personas con discapacidad participen de manera efectiva en una sociedad libre.

En este sentido, el proyecto PDICIENCIA se postula dentro de estos parámetros abordando los tres pilares de la educación inclusiva: la interacción de las personas con discapacidad intelectual o del desarrollo con los investigadores provoca que la transferencia del conocimiento sea más efectiva, ya que los científicos hacen inclusiva la explicación de sus investigaciones, fomentando el interés de las personas que participan de la divulgación. En cuanto a los obstáculos encontrados en su desarrollo:

- Se trabaja con un grupo de personas con discapacidad intelectual en un proyecto de divulgación científica donde los temas abordados generalmente suelen ser de difícil comprensión incluso para personas sin discapacidad. De hecho, las habilidades de lectoescritura de los miembros de la muestra son muy limitadas, y solamente una persona del equipo sabe leer y escribir.

- En otras ocasiones, el proyecto se ha encontrado con el velado obstáculo de la incredulidad: de qué forma es posible transmitir con rigor el resultado de investigaciones científicas DESDE la discapacidad y no PARA la discapacidad.

- Y el principal obstáculo encontrado es el económico: se trata de una iniciativa desarrollada por una asociación con escasos fondos que, en buena parte, se ha autofinanciado y llevado a cabo a través de la colaboración desinteresada y voluntaria de múltiples personas.

Con respecto al primer obstáculo, se abordó adaptando los contenidos científicos a lectura fácil, trabajando en habilidades sociales y actividades de refuerzo de memoria de las personas con discapacidad que no sabían leer ni escribir, apostando también por mostrar su naturalidad en los programas, utilizando el humor como nexo para todo. Los resultados hablan por sí solos: todas las personas sin lectoescritura del equipo se inscribieron durante 2018 en el Centro de Adultos de la localidad para aprender a leer y escribir, y así adquirir competencias para seguir trabajando y avanzando en el proyecto.

11 Artículo 24. Educación. Convención sobre los Derechos de las personas con discapacidad. Recuperado de: https://www.boe.es/boe/dias/2008/04/21/pdfs/A20648-20659.pdf 
En el caso del segundo obstáculo, apostando por normalizar la interacción entre científicos, investigadores y personas con discapacidad intelectual, para derribar esas barreras que responden solamente a clichés sociales.

En cuanto al tercer obstáculo, el proyecto fue presentado a diversas convocatorias de premios y subvenciones, logrando financiación para su ejecución. Las acciones del proyecto PDICIENCIA han supuesto la participación en numerosos eventos, así como el reconocimiento por parte de prestigiosas entidades en el ámbito de la innovación, la inclusión y la comunicación científica ${ }^{12}$.

\subsection{Creatividad, cine y discapacidad intelectual}

El agente de innovación local (AIL) del Centro Guadalinfo de Bailén (Jaén) colabora desde 2010 en la alfabetización e inclusión digital de las personas con discapacidad intelectual, como herramienta para mejorar su CDV, anticipándose en más de una década a los postulados que recoge la Asociación Somos Digital sobre las necesidades digitales de los beneficiarios de estas actividades, publicados en su estudio sobre los Centros de Competencias Digitales del Futuro (2020). En 2015, la red andaluza de centros Guadalinfo, de la que forma parte el AIL, comenzó a capacitar como expertos en innovación social a sus agentes dinamizadores ${ }^{13}$. A partir de entonces, se testearon en la Residencia y CDO Pedro Gámez diversas metodologías innovadoras relacionadas con la comunicación y desarrollo de proyectos de innovación, la búsqueda de fórmulas de financiación alternativas y talleres de pensamiento creativo o design thinking. Este conjunto de acciones, además, pretendía aportar contenido racional al tiempo libre de las personas con discapacidad intelectual, mediante el aprendizaje de herramientas informáticas y tecnológicas o el desarrollo de talleres de creatividad. Todas las áreas se plantearon para que tuvieran relación con temas de su interés, de modo que les estimulasen, y fuesen de carácter lúdico y didáctico-educativo. Se implementaron asimismo una serie de talleres de producción cinematográfica en los que las personas con discapacidad se familiarizaron con las herramientas y técnicas necesarias para llevar a cabo proyectos audiovisuales de muy diversa índole, desde cortometrajes hasta reportajes documentales o vídeos de sensibilización y concienciación sobre la discapacidad. Fomentando la participación de estas personas en los recursos de la localidad y su entorno, se involucró a este colectivo en la búsqueda de soluciones creativas a sus problemas, apostando por su plena inclusión y la visibilización de sus capacidades ante la sociedad.

No solo se pretendía evitar el aislamiento de las personas con discapacidad intelectual, sino, también, potenciar sus habilidades sociales y sus capacidades. El objetivo fundamental era transitar de la divergencia a la convergencia, pasando por un proceso

12 Véase https://www.pdiciencia.com/premios-y-eventos/

13 Consorcio Fernando de los Ríos (2018). Plan Operativo Anual de la Red Guadalinfo. Informe de Gestión. Página 7. Recuperado de http://www.guadalinfo.es/tenemosunplanparati/wp-content/ uploads/2018/10/Plan-Operativo-Anual-2018-v00_r04.pdf 
de síntesis y de análisis, para alcanzar la creatividad y la innovación, creando alternativas para la toma de decisiones (De Bono, 1994).

\subsection{Objetivos}

Tratamos de conocer la valoración y los beneficios de la participación de las personas con discapacidad intelectual en la realización de las actividades creativas, de producción cinematográfica y PDICIENCIA en el marco de actividades de ocio y tiempo libre incluidas en los planes de atención personalizados de la Residencia y CDO Pedro Gámez.

\section{Metodología}

El estudio tiene un carácter descriptivo, acerca de una experiencia de ocio en un centro residencial para personas con discapacidad intelectual. Partiendo de una perspectiva holística, y utilizando metodologías cualitativas, pretende describir las prácticas innovadoras desarrolladas a lo largo de un período de cinco años. Se han utilizado herramientas tales como cuestionarios de satisfacción y se ha realizado la medición de 5 de las 8 dimensiones de CDV de Schalock y Verdugo (2007) de las personas participantes, al estar más relacionadas con las actividades objeto de estudio: bienestar emocional, inclusión social, desarrollo personal, autodeterminación y relaciones interpersonales.

\subsection{Participantes}

La experiencia se ha desarrollado en la Residencia y Centro de Día Ocupacional Pedro Gámez de Bailén (Jaén) y ha contado con la participación de 10 personas con discapacidad intelectual (4 hombres y 6 mujeres), con grados de dependencia similares, que mantuvieron contacto diario con los profesionales implicados en el desarrollo de este estudio y recibieron su atención directa.

Los criterios de inclusión de las personas fueron los siguientes:

1. Haber participado en, al menos, 2 de las siguientes actividades: actividades de creatividad, de producción cinematográfica y PDICIENCIA.

2. Haber sido evaluadas en el ICV general entre 2015 y 2019.

3. Haber sido evaluadas en satisfacción con las actividades de ocio ofertadas en AFAMP en 2017, 2018 y 2019, y en PDICIENCIA en 2019.

\subsection{Procedimiento}

En los talleres de creatividad se implementaron las técnicas de creatividad más habituales (brainstorming, sombreros de pensar, muros de post-it, SCAMPER, mapas 
mentales...), a través de dinámicas de grupo enfocadas a la realización de proyectos de innovación social que tuviesen repercusión en la mejora de su CDV, así como en su desarrollo educativo. Asimismo, se trabajó en habilidades comunicativas, de integración e inclusión; en actividades compensatorias y de refuerzo intelectual, a través del trabajo con la inteligencia adaptativa, la memoria, y las habilidades sociales, orientando los talleres a la generación de ideas y el desarrollo de la creatividad para generar proyectos innovadores.

Enmarcados dentro de sus actividades de ocio y tiempo libre, los talleres de creatividad fueron evolucionando hacia acciones innovadoras con metodologías de Design Thinking (Stickdorn et al., 2011), centradas en entender y dar solución a las necesidades reales de las personas con discapacidad intelectual, y relacionadas con el fomento de su creatividad mediante la realización de actividades artísticas, técnicas de creatividad y el manejo de tecnologías de la información y la comunicación.

A partir de 2015, AFAMP puso en marcha un taller de producción cinematográfica, en el que se realizaron actividades formativas de carácter teórico-práctico (manejo de herramientas cinematográficas, adquisición de habilidades de interpretación). Se trabajó colectivamente en la confección de guiones y la generación de contenidos que, posteriormente, se utilizaron en los productos audiovisuales resultantes. "Lo que caracteriza al cine no es sólo la manera en que el hombre se presenta a sí mismo ante la cámara, sino también el modo en que, con la cámara, representa al mundo que lo rodea” (Benjamin, 2018, p. 45). Los objetivos del taller de producción cinematográfica y los de los talleres de creatividad y comunicación científica se vincularon como un laboratorio de ideas creativas cuyo epicentro ha sido la inclusión de las personas con discapacidad intelectual y su empoderamiento activo desde un ámbito innovador y disruptivo.

El equipo técnico (psicóloga y directora del CDO) de AFAMP realizó la selección de personas susceptibles de participar en este estudio en base a los criterios de inclusión citados en el apartado 3.1. El personal de atención directa se encargó de recabar la información sobre la satisfacción de las actividades de ocio y el responsable del proyecto PDICIENCIA se ha ocupado de medir la satisfacción de este proyecto concreto.

\subsection{Instrumentos de medida}

Para medir las actividades objeto de estudio se tuvieron en cuenta los registros de participación en actividades de ocio estable realizadas entre los años 2015 y 2019 (hasta el 31 de marzo). La evaluación del ICV general tiene carácter bianual, y se tuvieron en cuenta los períodos de 2015, 2017 y 2019. Una de las personas que participan en la muestra se incorporó a la residencia en 2016, por lo que no se cuenta con datos relativos al año 2015. Para evaluar la CDV de las personas, se han utilizado diferentes escalas de medida, según su perfil: la escala GENCAT (Verdugo et al., 2009), que realiza una evaluación objetiva, y la Escala INICO (Verdugo et al., 2013), que realiza una evaluación tanto objetiva como subjetiva. 
La evaluación en satisfacción con las actividades de ocio y tiempo libre de 2017, 2018 y 2019 se ha realizado a través de cuestionarios de satisfacción adaptados en lectura fácil y el específico de PDICIENCIA, en 2019.

\section{Resultados}

Las acciones de creatividad, producción cinematográfica y ciencia inclusiva (PDICIENCIA) en el marco de actividades de ocio y tiempo libre incluidas en los planes de atención personalizados de la Residencia y CDO Pedro Gámez han beneficiado a las personas que han participado en las mismas de múltiples formas. Les han permitido conocer a personas de otros ámbitos (científico, cinematográfico, artístico, etc.) que en ningún caso podrían haber conocido de otra manera. Además, han tenido acceso al ámbito de la ciencia y la cultura de una forma innovadora y fomentando su empoderamiento y protagonismo a través de su participación activa en eventos y actividades de diversa índole (véase nota 12), y en los resultados del cuestionario de satisfacción de PDICIENCIA en 2019 se aprecia un elevado índice de satisfacción (Tabla 2).

\begin{tabular}{|c|c|c|c|}
\hline \multirow[t]{2}{*}{ PREGUNTAS } & \multicolumn{3}{|c|}{ RESPUESTAS } \\
\hline & MUCHO & BASTANTE & POCO \\
\hline Me gusta participar en PDICIENCIA & $86,7 \%$ & $13,3 \%$ & \\
\hline Me interesa participar en las actividades que se proponen & $73,3 \%$ & $26,7 \%$ & \\
\hline $\begin{array}{l}\text { Me gusta la forma de trabajar de los responsables del } \\
\text { proyecto }\end{array}$ & $80 \%$ & $20 \%$ & \\
\hline La actividad se desarrolla como yo esperaba & $73,3 \%$ & $26,7 \%$ & \\
\hline He aprendido cosas que no sabía con esta actividad & $80 \%$ & $13,3 \%$ & $6,7 \%$ \\
\hline $\begin{array}{l}\text { Me gusta relacionarme con otras personas del mundo de } \\
\text { la ciencia }\end{array}$ & $86,7 \%$ & $13,3 \%$ & \\
\hline Mi valoración global de la actividad & $86,7 \%$ & $13,3 \%$ & \\
\hline
\end{tabular}

Fuente: Elaboración propia.

Los talleres de creatividad les han permitido relacionarse tanto en las actividades del centro como en externas, y trabajar con las ideas y las necesidades propias de las personas con discapacidad intelectual. En el caso de los talleres de producción cinematográfica, las personas participantes se han beneficiado formativamente en estos ámbitos, adquiriendo una serie de competencias que les han permitido realizar guiones, cortometrajes o documentales de concienciación sobre la discapacidad que se 
han podido exponer en diferentes foros e, incluso, conseguir importantes premios de carácter internacional ${ }^{14}$.

En cuanto a la Calidad de Vida, en 2015 se recogieron los primeros indicadores de CDV para 9 de las 10 personas. Se evaluaron en la escala GENCAT, situándose la media del ICV en el percentil 30,22. A partir del año 2017 se evaluó a cada persona según criterio del equipo técnico, en las escalas GENCAT (5 personas [2 $\hat{\jmath}, 3$ + $]$ ) e INICO (5 personas $[4 \hat{\jmath}, 1$ i $])$.

\begin{tabular}{|c|c|c|c|c|c|c|}
\hline \multirow{7}{*}{ Ui 42 ภิ } & Dimensiones & $2015 \mathrm{G}^{\mathrm{g}}$ & $2017 \mathrm{IOP}^{\mathrm{h}}$ & 2017 IAI $^{\mathrm{i}}$ & $2019 \mathrm{IOP}^{\mathrm{h}}$ & 2019 IAI $^{\mathrm{i}}$ \\
\hline & $\mathrm{BE}^{\mathrm{a}}$ & 50 & 63 & 40 & 63 & 75 \\
\hline & $\mathrm{DP}^{\mathrm{b}}$ & 25 & 25 & 25 & 37 & 50 \\
\hline & $\mathrm{A}^{\mathrm{c}}$ & 16 & 50 & 63 & 84 & 84 \\
\hline & $\mathrm{RI}^{\mathrm{d}}$ & 25 & 37 & 50 & 63 & 50 \\
\hline & $\mathrm{IS}^{\mathrm{e}}$ & 25 & 37 & 75 & 84 & 63 \\
\hline & $\mathrm{P}_{\mathrm{i}} \mathrm{ICV}^{\mathrm{f}}$ & 25 & 33 & 43 & 73 & 69 \\
\hline \multirow{7}{*}{ 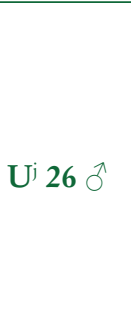 } & Dimensiones & $2015 \mathrm{G}^{\mathrm{g}}$ & $2017 \mathrm{IOP}^{\mathrm{h}}$ & 2017 IAI $^{\mathrm{i}}$ & $2019 \mathrm{IOP}^{\mathrm{h}}$ & 2019 IAI $^{\mathrm{i}}$ \\
\hline & $\mathrm{BE}^{\mathrm{a}}$ & 55 & 50 & 25 & 63 & 63 \\
\hline & $\mathrm{DP}^{\mathrm{b}}$ & 30 & 16 & 12 & 16 & 25 \\
\hline & $\mathrm{A}^{\mathrm{c}}$ & 25 & 25 & 37 & 75 & 63 \\
\hline & $\mathrm{RI}^{\mathrm{d}}$ & 16 & 25 & 9 & 25 & 25 \\
\hline & $\mathrm{IS}^{\mathrm{e}}$ & 11 & 16 & 16 & 50 & 50 \\
\hline & $\mathrm{P}_{\mathrm{i}} \mathrm{ICV}^{\mathrm{f}}$ & 17 & 17 & 8 & 46 & 50 \\
\hline \multirow{7}{*}{ Ui 121 ఠ } & Dimensiones & $2015 \mathrm{G}^{\mathrm{g}}$ & $2017 \mathrm{IOP}^{\mathrm{h}}$ & 2017 IAI $^{\mathrm{i}}$ & $2019 \mathrm{IOP}^{\mathrm{h}}$ & 2019 IAI $^{\mathrm{i}}$ \\
\hline & $\mathrm{BE}^{\mathrm{a}}$ & 75 & 75 & 50 & 84 & 75 \\
\hline & $\mathrm{DP}^{\mathrm{b}}$ & 25 & 25 & 25 & 50 & 37 \\
\hline & $\mathrm{A}^{\mathrm{c}}$ & 9 & 25 & 37 & 84 & 75 \\
\hline & $\mathrm{RI}^{\mathrm{d}}$ & 37 & 16 & 25 & 63 & 50 \\
\hline & $\mathrm{IS}^{\mathrm{e}}$ & 25 & 37 & 50 & 50 & 75 \\
\hline & $\mathrm{P}_{\mathrm{i}} \mathrm{ICV}^{\mathrm{f}}$ & 16 & 39 & 40 & 75 & 77 \\
\hline \multirow{7}{*}{ Ui 24} & Dimensiones & $2015 \mathrm{G}^{\mathrm{g}}$ & $2017 \mathrm{IOP}^{\mathrm{h}}$ & 2017 IAI $^{\mathrm{i}}$ & $2019 \mathrm{IOP}^{\mathrm{h}}$ & 2019 IAI $^{\mathrm{i}}$ \\
\hline & $\mathrm{BE}^{\mathrm{a}}$ & 10 & 5 & 5 & 16 & 16 \\
\hline & $\mathrm{DP}^{\mathrm{b}}$ & 16 & 19 & 25 & 50 & 50 \\
\hline & $\mathrm{A}^{\mathrm{c}}$ & 19 & 25 & 25 & 50 & 50 \\
\hline & $\mathrm{RI}^{\mathrm{d}}$ & 15 & 5 & 10 & 9 & 16 \\
\hline & $\mathrm{IS}^{\mathrm{e}}$ & 6 & 5 & 17 & 16 & 63 \\
\hline & $\mathrm{P}_{\mathrm{i}} \mathrm{ICV}^{\mathrm{f}}$ & 11 & 10 & 13 & 39 & 52 \\
\hline
\end{tabular}

14 En 2019, AFAMP recibió el premio al mejor cortometraje en la categoría "Ponte en nuestra piel" del INCLÙS, Festival Internacional de Cine y Discapacidad de Barcelona. http://www.inclus.cat/es/2019/ palm.html 
CIENCIA INCLUSIVA, CINE Y CREATIVIDAD: HERRAMIENTAS PARA MEJORAR

LA CALIDAD DE VIDA DE LAS PERSONAS CON DISCAPACIDAD INTELECTUAL

D. ORTEGA-ALONSO Y M. ${ }^{a}$ E. DE CASTRO-LÓPEZ

\begin{tabular}{|c|c|c|c|c|c|c|}
\hline \multirow{7}{*}{ Ui 131 우 } & Dimensiones & $2015 \mathrm{G}^{\mathrm{g}}$ & $2017 \mathrm{IOP}^{\mathrm{h}}$ & 2017 IAI $^{\mathrm{i}}$ & $2019 \mathrm{IOP}^{\mathrm{h}}$ & 2019 IAI $^{\mathrm{i}}$ \\
\hline & $\mathrm{BE}^{\mathrm{a}}$ & \multirow{6}{*}{ No estaba } & 63 & 37 & 63 & 63 \\
\hline & $\mathrm{DP}^{\mathrm{b}}$ & & 16 & 16 & 50 & 50 \\
\hline & $\mathrm{A}^{\mathrm{c}}$ & & 37 & 37 & 63 & 50 \\
\hline & $\mathrm{RI}^{\mathrm{d}}$ & & 25 & 16 & 50 & 25 \\
\hline & $\mathrm{IS}^{\mathrm{e}}$ & & 37 & 25 & 50 & 50 \\
\hline & $\mathrm{P}_{\mathrm{i}} \mathrm{ICV}^{\mathrm{f}}$ & & 31 & 29 & 59 & 55 \\
\hline
\end{tabular}

Abreviaciones: $\mathrm{BE}(\mathrm{a})=$ Bienestar emocional; DP $(\mathrm{b})=$ Desarrollo personal; A $(\mathrm{c})=$ Autodeterminación; RI $(\mathrm{d})=$ Relaciones interpersonales; IS $(\mathrm{e})=$ Inclusión Social; $\mathrm{P}_{\mathrm{i}} \mathrm{ICV}(\mathrm{f})=$ Percentil del Índice de Calidad de Vida; $\mathrm{G}(\mathrm{g})$ = GENCAT; IOP $(\mathrm{h})=$ INICO otras personas; IAI $(\mathrm{i})=$ INICO autoinforme; $\mathrm{U}(\mathrm{j})=$ Usuario/a. Elaboración propia.

\begin{tabular}{|c|c|c|c|c|}
\hline \multicolumn{5}{|c|}{ TABLA 3B. Resultados ICV (percentiles). GENCAT $(2015,2017,2019)$} \\
\hline \multirow{7}{*}{$\mathrm{U}^{\mathrm{i}} \mathbf{1 1 2}+$} & Dimensiones & $2015 \mathrm{G}^{\mathrm{g}}$ & $2017 \mathrm{G}^{\mathrm{g}}$ & $2019 \mathrm{G}^{\mathrm{g}}$ \\
\hline & $\mathrm{BE}^{\mathrm{a}}$ & 84 & 84 & 84 \\
\hline & $\mathrm{DP}^{\mathrm{b}}$ & 60 & 75 & 84 \\
\hline & $\mathrm{A}^{\mathrm{c}}$ & 25 & 26 & 63 \\
\hline & $\mathrm{RI}^{\mathrm{d}}$ & 65 & 70 & 75 \\
\hline & $\mathrm{IS}^{\mathrm{e}}$ & 75 & 80 & 84 \\
\hline & $\mathrm{P}_{\mathrm{i}} \mathrm{ICV}^{\mathrm{f}}$ & 53 & 60 & 77 \\
\hline \multirow{7}{*}{ U' 107 } & Dimensiones & $2015 \mathrm{G}^{\mathrm{g}}$ & $2017 \mathrm{G}^{\mathrm{g}}$ & $2019 \mathrm{G}^{\mathrm{g}}$ \\
\hline & $\mathrm{BE}^{\mathrm{a}}$ & 50 & 75 & 75 \\
\hline & $\mathrm{DP}^{\mathrm{b}}$ & 50 & 63 & 84 \\
\hline & $A^{c}$ & 16 & 16 & 63 \\
\hline & $\mathrm{RI}^{\mathrm{d}}$ & 5 & 5 & 16 \\
\hline & $\mathrm{IS}^{\mathrm{e}}$ & 12 & 16 & 50 \\
\hline & $\mathrm{P}_{\mathrm{i}} \mathrm{ICV}^{\mathrm{f}}$ & 14 & 29 & 60 \\
\hline \multirow{7}{*}{$\mathrm{U}^{\mathrm{i}} 115$} & Dimensiones & $2015 \mathrm{G}^{\mathrm{g}}$ & $2017 \mathrm{G}^{\mathrm{g}}$ & $2019 \mathrm{G}^{\mathrm{g}}$ \\
\hline & $\mathrm{BE}^{\mathrm{a}}$ & 84 & 84 & 84 \\
\hline & $\mathrm{DP}^{\mathrm{b}}$ & 63 & 75 & 95 \\
\hline & $\mathrm{A}^{\mathrm{c}}$ & 16 & 16 & 63 \\
\hline & $\mathrm{RI}^{\mathrm{d}}$ & 50 & 16 & 37 \\
\hline & $\mathrm{IS}^{\mathrm{e}}$ & 37 & 25 & 50 \\
\hline & $\mathrm{P}_{\mathrm{i}} \mathrm{ICV}^{\mathrm{f}}$ & 38 & 48 & 81 \\
\hline \multirow{7}{*}{$U^{j} 111$ ㅇ } & Dimensiones & $2015 \mathrm{G}^{\mathrm{g}}$ & $2017 \mathrm{G}^{\mathrm{g}}$ & $2019 \mathrm{G}^{\mathrm{g}}$ \\
\hline & $\mathrm{BE}^{\mathrm{a}}$ & 90 & 91 & 84 \\
\hline & $\mathrm{DP}^{\mathrm{b}}$ & 50 & 37 & 84 \\
\hline & $\mathrm{A}^{\mathrm{c}}$ & 9 & 16 & 63 \\
\hline & $\mathrm{RI}^{\mathrm{d}}$ & 60 & 63 & 63 \\
\hline & IS $^{\mathrm{e}}$ & 33 & 37 & 63 \\
\hline & $\mathrm{P}_{\mathrm{i}} \mathrm{ICV}^{\mathrm{f}}$ & 50 & 57 & 75 \\
\hline
\end{tabular}

Ediciones Universidad de Salamanca / CC BY-NC-ND

Siglo Cero, vol. 52 (3), 2021, julio-septiembre, pp. 141-161 
CIENCIA INCLUSIVA, CINE Y CREATIVIDAD: HERRAMIENTAS PARA MEJORAR LA CALIDAD DE VIDA DE LAS PERSONAS CON DISCAPACIDAD INTELECTUAL D. ORTEGA-ALONSO Y M. ${ }^{\text {a }}$. DE CASTRO-LÓPEZ

\begin{tabular}{|c|c|c|c|c|}
\hline \multirow{4}{*}{} & Dimensiones & $2015 \mathrm{G}^{\mathrm{g}}$ & $\mathbf{2 0 1 7} \mathrm{G}^{\mathrm{g}}$ & $\mathbf{2 0 1 9}^{\mathrm{g}}$ \\
\cline { 2 - 5 } & $\mathrm{BE}^{\mathrm{a}}$ & 84 & 84 & 63 \\
\cline { 2 - 5 } & $\mathrm{DP}^{\mathrm{b}}$ & 50 & 50 & 84 \\
\cline { 2 - 5 } & $\mathrm{A}^{\mathrm{c}}$ & 9 & 16 & 50 \\
\cline { 2 - 5 } & $\mathrm{RI}^{\mathrm{d}}$ & 50 & 37 & 37 \\
\cline { 2 - 5 } & $\mathrm{IS}^{\mathrm{e}}$ & 50 & 37 & 63 \\
\cline { 2 - 5 } & $\mathrm{P}_{\mathrm{i}} \mathrm{ICV}^{\mathrm{f}}$ & 48 & 45 & 63 \\
\hline
\end{tabular}

Abreviaciones: $\mathrm{BE}(\mathrm{a})=$ Bienestar emocional; DP $(\mathrm{b})=$ Desarrollo personal; $\mathrm{A}(\mathrm{c})=$ Autodeterminación; RI (d) = Relaciones interpersonales; IS (e) = Inclusión Social; $\mathrm{P}_{\mathrm{i}}$ ICV $(\mathrm{f})=$ Percentil del Índice de Calidad de Vida; $\mathrm{G}(\mathrm{g})$ = GENCAT; U (j) = Usuario/a. Elaboración propia.

En cuanto al grado de satisfacción con las actividades de ocio y tiempo libre de los residentes, los datos disponibles corresponden al período 2017-2019. En 2017, el 80 \% del total están satisfechos con dichas actividades. En 2018 la cifra es del 87 \%, incrementándose hasta el $90 \%$ en el año 2019.

\section{Discusión}

Tal y como afirman diversos autores (Arellano Torres y Peralta López, 2016; Badia Corbella y Araújo de Melo, 2009), la Planificación Centrada en la Persona debe incluir la elección personal del estilo de vida de las personas con discapacidad intelectual y el fomento de un mayor grado de independencia y participación en la comunidad, manifestando sus preferencias y deseos y pudiendo realizar sus propias elecciones. Esto incluye el ocio de las personas con discapacidad intelectual, que debe orientarse hacia la mejora de su calidad de vida favoreciendo las oportunidades, sin limitar su actividad y procurando la toma de decisiones de estas personas en la elección de las mismas. Las personas que se incluyen en las actividades objeto de estudio han elegido participar voluntariamente en las mismas, según sus propias preferencias y propuestas.

Se observa que todas las personas con discapacidad intelectual del presente estudio, en términos generales, han experimentado un incremento en CDV en el período comprendido entre 2015 y 2019, coincidiendo con su participación en actividades de ocio innovadoras. Pese a que intuimos que existe una relación entre ambos factores, no podemos concluir que este cambio ascendente sea exclusivamente atribuible a su intervención en las mismas, ya que no disponemos en este estudio de suficientes elementos relacionales, ni datos previos a 2015 de estas personas en materia de CDV. Además, existen una serie de limitaciones que deben tenerse en cuenta a la hora de interpretar los datos alcanzados. La escasez de bibliografía relacionada con la aplicación de metodologías de innovación social al ámbito de la discapacidad intelectual es una de ellas, y ha impedido apoyarnos en el diseño de instrumentos de medida en base a fuentes. Otra posible limitación del estudio es la referida a la utilización de 
dos escalas diferentes en los instrumentos de medida del ICV general, como son las escalas GENCAT e INICO. La elección de las mismas se realizó en base a las capacidades de cada persona evaluada según el criterio del equipo técnico de AFAMP. Por último, durante estos cinco años se ha dado principal importancia a la participación y a la toma de decisiones de las personas a la hora de dar forma a su proyecto de vida, por lo que se hace difícil atribuir los índices obtenidos en CDV exclusivamente a los factores planteados. No obstante, este estudio puede ser el punto de partida para el establecimiento de nuevas líneas de investigación que nos permitan indagar en la posible existencia de una correlación.

\subsection{Conclusiones}

Los planteamientos de trabajo de AFAMP en materia de CDV han ido evolucionando desde el año 2015. Anteriormente, las actividades de ocio para personas con discapacidad intelectual eran ofertadas desde la asociación y se asignaban en función del criterio de los profesionales, pero sus destinatarios no participaban en la toma de decisiones relacionadas con la elección de las mismas. A partir de esa fecha, las personas comenzaron a ser partícipes en esa toma de decisiones en materia de ocio, instaurándose como norma la oportunidad de escoger entre aquellas actividades que les fueran más interesantes en función de sus gustos, preferencias y deseos, y se empezaron a generar alternativas de ocio diferentes de las convencionales, algunas de las cuales fueron propuestas por las personas con discapacidad intelectual o consecuencia de las actividades que ya estaban en marcha.

La implementación de talleres de ocio fundamentados en la innovación social y la creatividad para las personas con discapacidad intelectual coincide temporalmente con este período, así como con el seguimiento desarrollado desde AFAMP sobre las metodologías basadas en el Modelo de CDV Individual de Schalock y Verdugo (2007), las metodologías de apoyo conductual positivo, apoyo activo, el modelo de transformación de servicios y la Planificación Centrada en la Persona.

En el caso concreto del proyecto PDICIENCIA, la interacción de las personas con discapacidad intelectual o del desarrollo con investigadores y científicos provoca que la transferencia del conocimiento sea más efectiva, ya que la explicación de sus investigaciones es inclusiva, fomentando el interés de las personas que participan de la divulgación y haciéndola más sencilla de entender en términos generales. El proyecto nos sitúa ante la tesitura de que, cuando la innovación social actúa como semillero de ideas, las capacidades intelectuales de las personas involucradas no son tan relevantes como su actitud, su resiliencia o el resultado de aplicar el pensamiento lateral (De Bono, 1991). De esta forma, las personas con discapacidad intelectual se involucran en procesos creativos que ayudan a hacer más accesible la información tanto en las asociaciones como en su entorno.

Los resultados nos invitan a reflexionar sobre la relevancia de incluir estrategias que apuesten por el desarrollo de la creatividad de las personas. El potencial que se genera ante el ofrecimiento de apoyos y oportunidades necesarios por parte de las 
asociaciones e instituciones puede servir para que las personas con discapacidad y sus familias puedan desarrollar una vida plena, apostando por su inclusión en todos los ámbitos.

\section{Agradecimientos}

La redacción de este artículo no habría sido posible sin la colaboración de Concepción Fernández Soriano, trabajadora social; Rosario Muñoz Moriana, psicóloga, y Antonio Padilla Cabrera, responsable de comunicación, así como del resto del equipo humano de AFAMP y del proyecto PDICIENCIA. También queremos agradecer la desinteresada ayuda de las doctoras María Martínez Morales, María Isabel Moreno Montoro y Eva Sotomayor Morales, quienes con sus aportaciones y comentarios han permitido enriquecer el texto y los contenidos del mismo. Desde estas líneas queremos expresar nuestra gratitud. Asimismo, queremos dedicar este artículo a Ramón Ruvira, el hijo del Lolo, un ser humano excepcional y maravilloso, a quien echaremos mucho de menos. De los errores o contrariedades que pudieran observarse en el presente artículo, asumimos nuestra entera responsabilidad.

\section{Referencias bibliográficas}

Aladro Vico, E., Cavadas Gormaz, M. J., Jivkova Semova, D., Padilla Castillo, G., Sosa SÁnchez, R. P. y Requejo Rey, P. (2018). Artivismo como herramienta de transformación social: dos iniciativas en los barrios de La Latina y Lavapiés (Madrid) 1. Comunitania, 15, 171-196. https://doi:10.5944/comunitania.15.9

Alonso, D. (2018). Desarrollo de las habilidades motrices de las personas con discapacidad intelectual a través del proceso cognitivo. Artseduca, 19, 224-245. https://doi:10.6035/Artseduca.2018.19.10

Alonso, M. J. y De Araoz, I. (2011). El impacto de la Convención Internacional sobre los Derechos de las Personas con Discapacidad en la legislación educativa española. CERMI.

Alonso-Flores, J., Serrano-López, A. E. y Moreno-Castro, C. (2018). La publicación de noticias sobre los resultados de I+D+i. ¿Cómo es percibida por los investigadores españoles? InMediaciones de la Comunicación, 13(2), 115-140. https://doi:10.18861/ic.2018.13.2.2870

Arellano Torres, A. y Peralta lópez, F. (2016). La planificación centrada en la persona: un ejemplo de buena práctica en el ámbito de la discapacidad intelectual. Contextos Educativos. Revista de Educación, O(19), 195-212. https://doi.org/10.18172/con.2754

Arias, B., Irurtia, M. J. y Gómez, L. E. (2009). Conductas problemáticas en personas con discapacidad intelectual: un estudio preliminar sobre dimensionalidad y propuesta de clasificación. Psicología Conductual, 17(2), 257-275.

Asociación de Familiares y Amigos de Personas con Discapacidad Intelectual. (2018a). Memoria de actividades de AFAMP 2018. Recuperado de: https://www.afamp.org/ con $\% \mathrm{C} 3 \% \mathrm{~B} 3$ cenos/transparencia/ 
CIENCIA INCLUSIVA, CINE Y CREATIVIDAD: HERRAMIENTAS PARA MEJORAR

LA CALIDAD DE VIDA DE LAS PERSONAS CON DISCAPACIDAD INTELECTUAL

D. ORTEGA-ALONSO Y M. ${ }^{\text {a }}$ E. DE CASTRO-LÓPEZ

Asociación de Familiares y Amigos de Personas con Discapacidad Intelectual. (2018b). Memoria Residencia Pedro Gámez 2018. Recuperado de: https://www.afamp.org/ con $\% \mathrm{C} 3 \% \mathrm{~B} 3$ cenos/transparencia/

Asociación de Familiares y Amigos de Personas con Discapacidad Intelectual. (2019a). Plan Estratégico AFAMP 2019-2021. Recuperado de: https://www.afamp.org/ con $\% \mathrm{C} 3 \% \mathrm{~B} 3$ cenos/transparencia/

Asociación de Familiares y Amigos de Personas con Discapacidad Intelectual. (2019b). Programación General Residencia Pedro Gámez 2019. Recuperado de: https://www.afamp. org/con\% C3\%B3cenos/transparencia/

Badia Corbella, M. y Longo Araújo de Melo, E. (2009). El ocio en las personas con discapacidad intelectual: participación y calidad de vida a través de las actividades de ocio. Siglo Cero, $40(3), 30-44$.

Ballesta, A. M., Vizcaíno, O. y Mesas, E. C. (2011). El Arte como un lenguaje posible en las personas con capacidades diversas. Arte y Políticas de Identidad, 4, 137-152. Recuperado de https://revistas.um.es/reapi/article/view/146051

Bank-Mikkelsen, N. (1975). El principio de normalización. Siglo Cero, 37, 16-21.

Benjamin, W. (2018). La obra de arte en la época de su reproducción mecánica. Casimiro.

Buzan, T. y Buzan, B. (1996). El libro de los mapas mentales. Ediciones Urano.

CAmpos DE CAmpos, D. (1995). La expresión plástica en las personas con sindrome de Down en la Comunidad de Madrid (Tesis doctoral). Recuperado de http://hdl.handle.net/11162/41763

Cárdenas, R., BArriga, A. y Lizama, J. (2017). La expresión artística como estrategia didáctica para el desarrollo de la afectividad y la autoestima en una persona con discapacidad intelectual y Síndrome Alcohólico Fetal (SAF). Arte, Individuo y Sociedad, 29(3), 205-222. https://doi:10.5209/ARIS.53623

Carratalá Marco, A., Mata Roig, G. y Crespo Varela, S. (2017). Planificación centrada en la persona: planificando por adelantado el futuro deseado. Plena inclusión España. Recuperado de http://hdl.handle.net/11181/5298

Centro de Documentación y Estudios SiIS Dokumentazio eta Ikerketa Zentroa. (2011a). Vivir mejor. Buenas prácticas en la atención a personas con discapacidad. Apoyo conductual positivo. Fundación Eguía-Careaga Fundazioa. Diputación Foral de Álava.

Centro de Documentación y Estudios SiIS Dokumentazio eta Ikerketa Zentroa. (2011b). Vivir mejor. Buenas prácticas en la atención a personas con discapacidad. Planificación esencial del estilo de vida. Fundación Eguía-Careaga Fundazioa. Diputación Foral de Álava.

Centro de Documentación y Estudios SiIS Dokumentazio eta Ikerketa Zentroa. (2011c). Vivir mejor. Cómo concebimos la atención residencial. Pautas básicas para una atención de calidad a las personas con discapacidad. Fundación Eguía-Careaga Fundazioa. Diputación Foral de Álava.

Christensen, C. M., Raynor, M. E. y McDonald, R. (2015). What is disruptive innovation. Harvard Business Review, 93(12), 44-53.

Colmenero Ruiz, M. J. (2015). Caminando hacia una educación inclusiva. Caminando hacia una sociedad inclusiva. Iniciación a la Investigación, 1. Recuperado de https://revistaselectronicas.ujaen.es/index.php/ininv/article/view/2554

Consorcio Fernando de los Ríos. (2018). Plan Operativo Anual de la Red Guadalinfo. Informe de Gestión. Recuperado de: http://consorciofernandodelosrios.es/descargas/Informe_POA_2018.pdf

De Bono, E. (1991). Pensamiento lateral. Paidós.

De Bono, E. y DiéGuez, R. D. (1988). Seis sombreros para pensar. Juan Granica.

Ediciones Universidad de Salamanca / CC BY-NC-ND

Siglo Cero, vol. 52 (3), 2021, julio-septiembre, pp. 141-161 
CIENCIA INCLUSIVA, CINE Y CREATIVIDAD: HERRAMIENTAS PARA MEJORAR

LA CALIDAD DE VIDA DE LAS PERSONAS CON DISCAPACIDAD INTELECTUAL

D. ORTEGA-ALONSO Y M. ${ }^{\text {a }}$ E. DE CASTRO-LÓPEZ

De Bono, E. y Castillo, O. (1994). El pensamiento creativo. Paidós.

Diehl, M. y Stroebe, W. (1987). Productivity loss in brainstorming groups: toward the solution of a riddle. Journal of Personality and Social Psychology, 53(3), 497.

Eberle, B. (1996). Scamper on: games for imagination development. Prufrock Press Inc.

Echevarría, J. (2008). El manual de Oslo y la innovación social. Arbor, 184(732), 609-618. https://doi:10.3989/arbor.2008.i732.210

ETXEBERRÍA, X. (2008). La condición de ciudadanía de las personas con discapacidad intelectual. Universidad de Deusto. Recuperado de http://www.deusto-publicaciones.es/deusto/pdfs/ cuadernosdcho/cuadernosdcho48.pdf

European Comission. (2016). Open Innovation. Open Science, Open to the World - a vision for Europe. Recuperado de: http://publications.europa.eu/resource/cellar/3213b3351cbc-11e6-ba9a-01aa75ed71a1.0001.02/DOC_2

Fundación Española para la Ciencia y la Tecnología. (2018). Informe de resultados de Percepción Social de la Ciencia y la Tecnología 2018. Recuperado de: https:/icono.fecyt.es/ informes-y-publicaciones/percepcion-social-de-la-ciencia-y-la-tecnologia-en-espana

GARCíA, E. (1988). La integración escolar: aspectos psicosociológicos. UNED.

García Ceballos, S. (2013). Educación artística para la inclusión social de personas con discapacidad intelectual (Trabajo Fin de Máster). Recuperado de: http://uvadoc.uva.es/handle/10324/2690

García-Frank, A., Canales, M. L., Gómez-Heras, M., González Acebrón, L., Hontecillas, D., Del Moral, B., Muñoz-García, M. B. y Sarmiento, G. N. (2013). Geodivulgar: una nueva experiencia para la divulgación de la Geología entre alumnos con necesidades educativas especiales por discapacidad intelectual. En I. RÁbANo y A. Rodrigo (Eds.), XX Bienal de la Real Sociedad Española de Historia Natural (pp. 124-125).

García-Frank, A., Gómez-Heras, M., Fesharaki, O., Iglesias Álvarez, N. y Gonzalo PaRRA, L. (2016). Science without barriers: towards the take-off of Social Palaeontology. Palaeontological Association Newsletter, 91, 50-55.

Gómez, L. E., Verdugo, M. Á., Arias, B. y Navas, P. (2008). Evaluación de la CDV en personas mayores y con discapacidad: la Escala FUMAT. Psychosocial Intervention, 17(2), 189-199.

Gómez González, F., Lacasta Reoyo, J., Martínez-Tur, V. y Rodríguez Sumaza, C. (2018). Avances en el liderazgo: marco de competencias de los líderes profesionales. Siglo Cero, 49(4), 7-34. https://doi:10.14201/scero2018494734

Goñi Garrido, M. J., Martínez Rueda, N. y Zardoya Santos, A. (2012). Apoyo conductual positivo. Algunas herramientas para afrontar las conductas difíciles. FEAPS. Recuperado de: http://hdl.handle.net/11181/3259

Hall, E. (2010). Spaces of social inclusion and belonging for people with intellectual disabilities. Journal of Intellectual Disability Research, 54, 48-57. https://doi:10.1111/j.13652788.2009.01237.x

Himanen, P. (2002). La ética del hacker y el espíritu de la era de la información. Recuperado de: http://eprints.rclis.org/12851/1/pekka.pdf

Iglesias Álvarez, N., Fesharaki, O., García-Frank, A., González Acebrón, L., Rico, R.,R., Salazar Ramírez, R. W., Sacristán, S., Martín Perea, D., Hontecillas, D., García Hernández, R., Gómez-Heras, M., Sarmiento, G. N., Muñoz García, B., Ureta Gil, S., Canales Fernández, M. L. y Del Moral, B. (2015). Dejando huella: divulgación paleontológica para personas con diversidad funcional. En L. Domingo et al. (Eds.), Current Trends in Paleontology and Evolution (pp. 154-162). XIII EJIP Conference Proceedings.

Ediciones Universidad de Salamanca / CC BY-NC-ND

Siglo Cero, vol. 52 (3), 2021, julio-septiembre, pp. 141-161 
CIENCIA INCLUSIVA, CINE Y CREATIVIDAD: HERRAMIENTAS PARA MEJORAR

LA CALIDAD DE VIDA DE LAS PERSONAS CON DISCAPACIDAD INTELECTUAL

D. ORTEGA-ALONSO Y M. ${ }^{\mathrm{a}}$ E. DE CASTRO-LÓPEZ

Jiménez, P. C., De Gracia, A. O., Ceballos, S. G., González, N. D., Vegas, S. G., RodríGuez, E. S. y SÁez, A. I. L. (2013). Arte para todos. Proyecto de investigación y creación con personas con capacidades diversas. Arteterapia, 8, 155-168. https://doi:10.5209/rev_ ARTE.2013.v8.44443

Lobato Suero, M. J., Martínez Pecino, M. y Molinos Lara, I. (2003). El desarrollo de habilidades en las personas con necesidades educativas especiales a través de la expresión plástica. EA, Escuela Abierta: Revista de Investigación Educativa, 6, 47-70.

López-Torrijo, M. (2009). La inclusión educativa de alumnos con discapacidades graves y permanentes en la Unión Europea. Relieve-Revista Electrónica de Investigación y Evaluación Educativa, 15(1), 1-20. Recuperado de: https://www.uv.es/RELIEVE/v15n1/RELIEVEv15n1_5.pdf

Martín, J. C. (2006). Modelo de Calidad de Vida de Schalock y Verdugo (2002-2003): validación mediante análisis multivariable. Tesis doctoral no publicada. Universidad de Salamanca.

Martínez-Tur, V, Peiró, J. M., Moliner C. y Potocnik, K. (2010). Calidad de servicio y CDV: el "survey feedback" como estrategia de cambio organizacional. Feaps.

Martínez-Tur, V. y Peñarroja, V. (2012). Innovación en el Tercer Sector ¿ Crisis de iniciativa? ICONG.

Martorell, V., Turrero, M., Calero, A. y Reyes, S. (2014). Participación y planes de vida. Estrategias de participación para las personas con grandes necesidades de apoyo. Grupo Fundosa Technosite. Fundación Once.

Matilde, F. E. R. y EnRíquez, C. I. D. (2010). Medios de comunicación, conformación de imagen y construcción de sentido en relación a la discapacidad. Política y Sociedad, 47(1), 105-113. Recuperado de: https://revistas.ucm.es/index.php/POSO/article/view/POSO1010130105A

Minayo, M. C. D. S., Deslandes, S. F., Neto, O. C. y Gomes, R. (1994). Teoría, método y creatividad. Lugar Editorial.

Moreno Montoro, M. y Martínez Morales, M. (2019). Hacer camino. InVisibilidades, 11, 154-157. https://doi:10.24981.16470508.11.19

Muñiz, F., Romero, A., Martínez, R., Durán, M. V., Narváez, M. J., Lozano, O. y Martín, M. (2018). Mineralogía con tacto. Enseñanza de las Ciencias de la Tierra, 26(3), 366-374. Recuperado de: https://www.raco.cat/index.php/ECT/article/view/343198

Muñoz-García, M. B., González-Acebrón, L., García-Frank, A., Pérez Barroso, R., Espín Forjan, B., Benito Manjón, P., De Pablo Gutiérrez, L., Gómez-Heras, M., Canales Fernández, M. L., Sarmiento Chiesa, G., Ureta Gil, S. y Del Moral González, B. (2015). Evaluación del aprendizaje significativo del concepto "tiempo geológico" en estudiantes con necesidades educativas especiales por discapacidad intelectual. Enseñanza de las Ciencias de la Tierra, 23(1), 220-231. Recuperado de: https://www.researchgate.net/ publication/301793895_Evaluacion_del_aprendizaje_significativo_del_concepto_Tiempo_Geologico_en_estudiantes_con_necesidades_educativas_especiales_por_discapacidad_ intelectual

Oficina del Alto Comisionado para los Derechos Humanos. ACNUDH. (1966). Discapacidad y protocolo facultativo. International Covenant on economic, social, and cultural rights. Recuperado de: https://www.ohchr.org/SP/ProfessionalInterest/Pages/CESCR. aspx 
CIENCIA INCLUSIVA, CINE Y CREATIVIDAD: HERRAMIENTAS PARA MEJORAR

LA CALIDAD DE VIDA DE LAS PERSONAS CON DISCAPACIDAD INTELECTUAL

D. ORTEGA-ALONSO Y M. ${ }^{\mathrm{a}}$ E. DE CASTRO-LÓPEZ

Organización de Naciones Unidas. (2008). Convención sobre los Derechos de las Personas con Ratificación de la Convención sobre los Derechos de las Personas con Discapacidad). Recuperado de: https://www.boe.es/boe/dias/2008/04/21/pdfs/A20648-20659.pdf

Organización de Naciones Unidas. (2015a). Agenda 2030 de Desarrollo Sostenible. Recuperado de: https://www.un.org/sustainabledevelopment/es/objetivos-de-desarrollo-sostenible/

Organización de Naciones Unidas. (2015b). Transformar nuestro mundo: Agenda 2030 de Desarrollo Sostenible. Recuperado de: https://www.un.org/en/ga/search/view_doc. asp?symbol=A/RES/70/1\&Lang=S

Palacios, A. (2008). El modelo social de discapacidad: orígenes, caracterización y plasmación en la Convención Internacional sobre los Derechos de las Personas con Discapacidad. CERMI.

Pallisera Díaz, M. (2013). La planificación centrada en la persona (PCP): una vía para la construcción de proyectos personalizados con personas con discapacidad intelectual. Revista Iberoamericana de Educación, 56(3), 1-12.

Pérez, D. C. (2004). Conocimiento y gestión del empleo de las personas con discapacidad. Revista del Ministerio de Trabajo y Asuntos Sociales.

Pérez-Montero, E. (2019). Towards a more inclusive outreach. Nature Astronomy, 3(2), $114-$ 115. https://doi:10.1038/s41550-019-0693-3

Plena inclusión (2016). V Plan Estratégico 2016-2020. Plena inclusión.

Rodríguez, I. (2017). Ciencia para Lia y Edmu: cinco experiencias creativas de divulgación científica. Trabajo presentado en el VI Congreso de Comunicación Social de la Ciencia, Córdoba.

Rodríguez, T. C., García, M. T. I. y Fernández, S. F. (2016). El apoyo activo como herramienta para la mejora de la participación de la persona con discapacidad intelectual. Estado de la cuestión. Revista Española de Discapacidad (REDIS), 4(2), 47-62. http:// doi:10.5569/2340-5104.04.02.03

SCHAlock, R. (2010). Aplicaciones del paradigma de calidad de vida a las personas con discapacidad intelectual y del desarrollo. En M. Á. Verdugo, M. Crespo y T. Nieto (Coords.), Aplicación del paradigma de calidad de vida. VII Seminario de Actualización Metodológica en Investigación sobre Discapacidad SAID (pp. 11-18) Publicaciones del INICO.

Schalock, R. y Verdugo, M. Á. (2007). El concepto de calidad de vida en los servicios y apoyos para personas con discapacidad intelectual. Siglo Cero, 38(4), 21-36.

Somos Digital. (2020). Los centros de competencias digitales del futuro. Junta de Castilla y León, Tecnalia. Recuperado de https://somos-digital.org/wp-content/uploads/2020/03/ Los-Centros-de-Competencias-Digitales-del-Futuro.pdf

Stickdorn, M., Schneider, J., ANdrews, K. y LAwrence, A. (2011). This is service design thinking: basics, tools, cases (vol. 1). Wiley.

TAMARIT, J. (2015). La transformación de los servicios hacia la calidad de vida. Una iniciativa de innovación social de FEAPS. Siglo Cero, 46(3), 47-71. https://doi:10.1352/1934-955650.5.403.

Verdugo, M. Á., Arias, B., Gómez, L. E. y Schalock, R. L. (2009). Formulario de la Escala GENCAT de calidad de vida. Manual de aplicación de la Escala GENCAT de calidad de vida. Departamento de Acción Social y Ciudadanía.

Verdugo, M. Á., Gómez, L. E., Arias, B., Santamaría, M., Clavero, D. y Tamarit, J. (2013). Escala INICO-FEAPS. Evaluación integral de la CDV de personas con discapacidad intelectual o del desarrollo. INICO.

Ediciones Universidad de Salamanca / CC BY-NC-ND

Siglo Cero, vol. 52 (3), 2021, julio-septiembre, pp. 141-161 


\section{CIENCIA INCLUSIVA, CINE Y CREATIVIDAD: HERRAMIENTAS PARA MEJORAR LA CALIDAD DE VIDA DE LAS PERSONAS CON DISCAPACIDAD INTELECTUAL D. ORTEGA-ALONSO Y M. ${ }^{a}$ E. DE CASTRO-LÓPEZ}

Wolfensberger, W. (1972). El principio de la normalización de los servicios humanos. Instituto Nacional de Retraso Mental.

Zambrano Valdivieso, O., Almeida Salinas, O., Rodríguez Serrano, K., Vargas Ramírez, I. y Silva PRADA, D. (2018). Innovación disruptiva, estrategia de inclusión al emprendimiento social. Inclusión y Desarrollo, 6(1), 33-46. https://doi:10.26620/uniminuto.inclusion.6.1.2019.33-46 


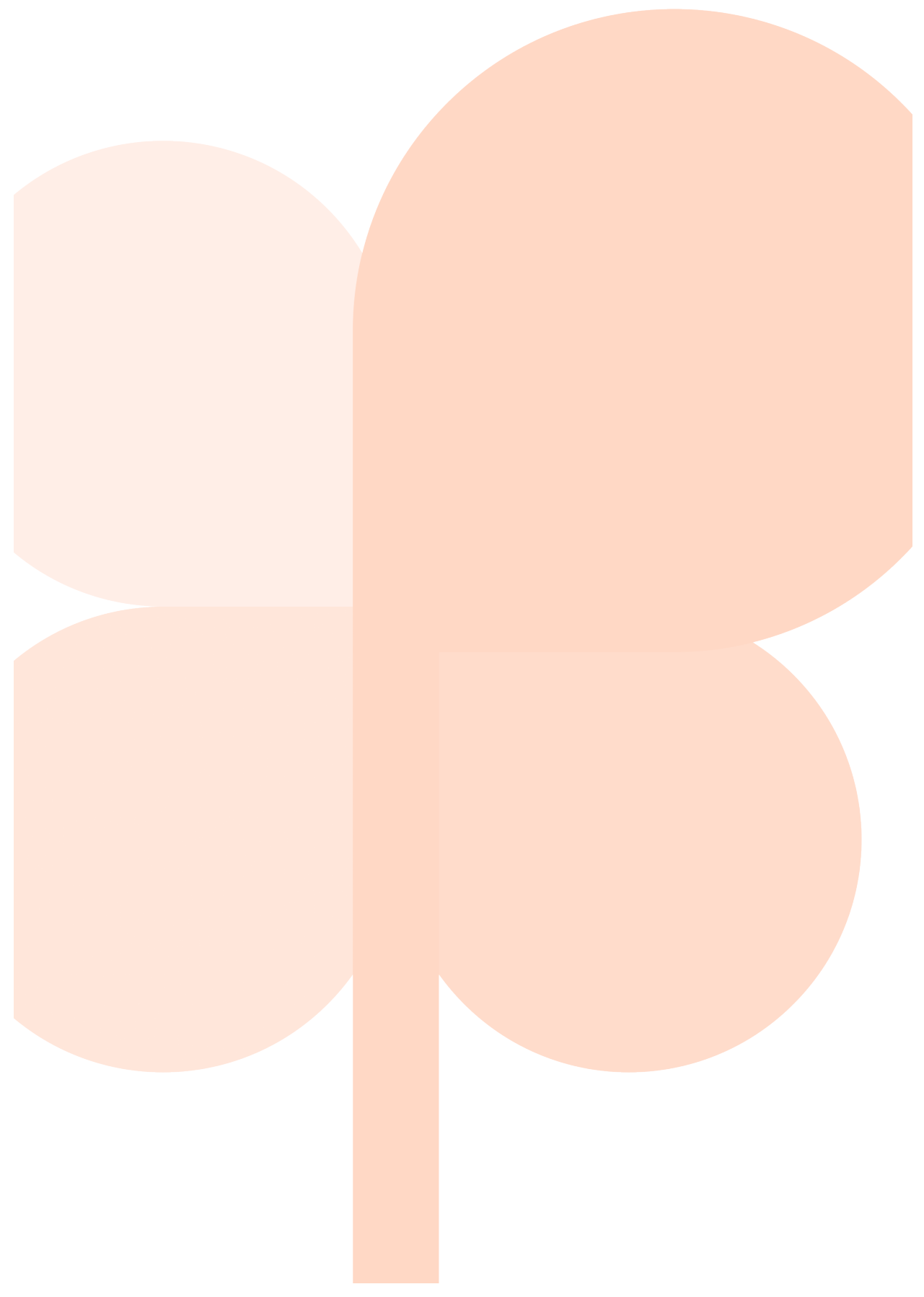

